\title{
Effect of Keratolytic Drugs on Oral Hyperkeratosis in Syrian Hamsters
}

\author{
JOHN A. CAPODANNO* and JAMES R. HAYWARD \\ University of Michigan Medical Center, Department of Oral Surgery, Ann Arbor, Michigan
}

\section{SYNOPSIS IN INTERLINGUA}

Le Effecto de Pharmacos Keratolytic Super Hyperkeratosis Oral.--Lesiones hyperkeratotic del mucosa oral esseva inducite in albin hamsters syrian per le administration topic de 9,10-dimethyl-1,2benzanthraceno. Post 6 applicationes de un solution de 20 pro cento de acido salicylic, un significative declino quantitative esseva observate clinicamente in le keratosis. Associate alterationes microscopic del processo de keratinisation esseva le formation de un amorphe, non-laminate strato cornee, perdita de strato lucide, e disruption del strato granulose. Mucosa hyperkeratotic tractate similemente con un solution de 20 pro cento de podophyllina manifestava nulle comparabilemente significative effecto keratolytic. Es stipulate que le inefficacia relative de podophyllina in le presente studio esseva conditionate per su supponite incapacitate de penetrar epithelio cornificate o per su inactivation therapeutic in le saliva alcalin del hamster.

Keratotic changes in the oral cavity have long been recognized as lesions of considerable significance that represent an important diagnostic and therapeutic responsibility of the dental profession. To date, no single systemic or local therapeutic regime has evolved that is consistently successful in the management of this condition. The problem is further complicated by the common occurrence of these lesions in people of advancing years, and the frequent involvement of multiple sites, encompassing large areas of oral mucosa. Although the geriatric patient has the physiologic ability to tolerate a local surgical excision, removal of extensive regions of the oral mucosa would be too traumatic. It would appear desirable, therefore, to develop an effective regime of eliminating oral hyperkeratosis without widespread loss of surface epithelium.

The utilization of keratolytic medicaments, long employed in the specialty of dermatology, ${ }^{1-4}$ appeared to offer a potentially effective means of treating this problem. The intraoral application of such agents, however, has been limited to the treatment of hairy tongue. ${ }^{5,6}$ Reports of

This study was conducted in the research facilities of the United States Veterans Administration Hospital, Ann Arbor,

Received for publication October 18, 1965.

* Present address: College of Dentistry, University of Kentucky Medical Center, Department of Oral Surgery, Lexing-
ton, Ky. their therapeutic use in treating other abnormal oral keratoses could not be found. Of the keratolytic drugs surveyed, podophyllin (podophyllum resin) and salicylic acid appeared to be the most effective and widely employed. A study seemed warranted, therefore, to investigate the therapeutic efficacy of podophyllum resin and salicylic acid in eradicating hyperkeratosis of oral mucous membranes.

\section{Materials and Methods}

To induce focal hyperkeratotic lesions of the hamster oral mucosa, a carcinogenic polycyclic hydrocarbon, 9,10-dimethyl-1,2benzanthracene (DMBA), $\uparrow$ was selected for use in this study. This drug was utilized in a 0.5 per cent solution, the optimal concentration capable of producing maximal tumor response without loss of animals due to toxicity. ${ }^{7}$ It was dissolved in mineral oil to effect a more intimate contact with the cells of the mucosal epithelium. ${ }^{8}$

Fifteen Syrian albino hamsters, 3 weeks of age, were used in the study. A modification of the pouch retracting device described by Morris $^{9}$ was employed, and the hydrocarbon was applied to the epithelial surfaces of the cheek pouches of unanesthetized hamsters with a No. 4 camel's hair brush. An estimated dose of $31.3 \mathrm{mg}$. of the carcinogen was applied topically to the

† Distiliation Products Division, Eastman Kodak Co., Rochester, N.Y. 
pouches once a week for 12 weeks. The hamsters had been allotted to four groups. In two of these groups, the cheek pouches were treated bilaterally with DMBA. In the other two groups, only the left pouches were treated. Clinical evidence of hyperkeratotic changes was observed by the 12 th week.

At the termination of the 12 -week period, the treated pouches were carefully examined and representative areas were delineated by tattoo markings subsequent to biopsy and photographic procedures. In the first group, consisting of 5 hamsters whose cheek pouches were treated bilaterally with the carcinogen, one pouch of each animal was selected for application of 20 per cent salicylic acid.* The second group, composed of 6 hamsters similarly pretreated, was selected for the unilateral application of 20 per cent podophyllin. $f$ In the final two groups of 2 hamsters each, with previous exposure of only one pouch to the hydrocarbon, the keratolytics were applied bilaterally. In one group, 20 per cent podophyllin was used, and in the other, 20 per cent salicylic acid was used.

The acute inflammatory response was permitted to subside during an 8-week period after the last application of DMBA. Then the application of the keratolytic agents was initiated. The drugs were suspended in compound tincture of benzoin $\ddagger$ and were applied topically to the designated areas with a No. 3 camel's hair brush. The compound tincture of benzoin vehicle permitted the medication to be applied accurately to the designated site without uncontrolled spread. ${ }^{10}$

The podophyllin and salicylic acid were applied weekly to the pouches of hamsters anesthetized with intraperitoneal injections of sodium pentobarbital $\$$ at a rate of 5 $\mathrm{mg} . / 100 \mathrm{Gm}$. of body weight. After the second application of the keratolytics, the inflammatory response was so acute that a period of 10 days was permitted to elapse before resuming treatment. On resumption, keratolytic applications were continued at weekly intervals until a total of six treat-

* Merck and Company, Inc, Rahway, N.J.

$\dagger$ Merck Sharp and Dohme, Division of Merck and Company, Inc., West Point, Pa.

$\ddagger$ The Wm. S. Merrell Company, Division of RichardsonMerrell Inc., Cincinnati, Ohio.

$\S$ Nembutal, Abbott Laboratories, North Chicago, Ill. ments had been given. At this time, in view of extensive inflammation, scarring, and neoplastic involvement of a number of pouches, application of the drugs was discontinued.

Gross observations and photographs were made of the treated areas of the mucosa and the corresponding control areas. Biopsy specimens from the areas then were obtained for microscopic examination.

\section{Results}

Gross observations.-Prior to the application of the respective keratolytic agents, the carcinogen-treated mucosa exhibited a slight but definite increase in keratinization. Moreover, multiple, small, elevated, papillomatous lesions involved the mucosa, particularly in the apical portion of the everted cheek pouch, which represents the most inferior point in the normal, resting oral cavity of the hamster. Although the lesions were small and discrete, their friable nature caused considerable bleeding in response to slight trauma. The untreated normal mucosa, however, demonstrated only slight evidence of surface keratinization and no indication of neoplastic involvement.

After the application of 20 per cent salicylic acid to the conditioned mucosa, the initial alterations observed grossly were desquamation, ulceration, and bleeding. As the program of keratolytic application progressed, the amount of surface hyperkeratosis decreased greatly but the number and size of the previously noted neoplastic lesions increased considerably. In addition to the neoplastic proliferation, the inflammatory response produced by salicylic acid on the carcinogen-treated mucosa increased significantly. In contrast, the mucosa of the control pouches, treated only with DMBA, lacked any obvious decrease in the degree of keratinization and did not show the accelerated neoplastic activity seen with salicylic acid application.

In the podophyllin-treated mucosa, no significant keratolytic effect was apparent clinically. In fact, in several instances, the surface keratinization of the treated zone appeared to increase. The proliferation of the previously observed lesions continued in a manner similar to that observed in the salicylic acid series. There was no apparent 


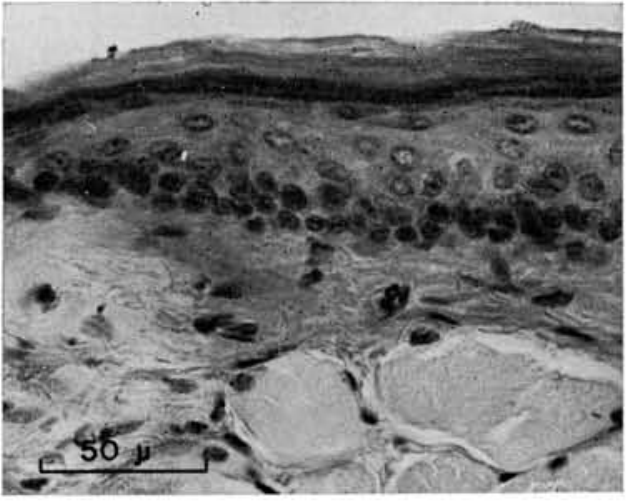

Fig. 1.-Hamster oral mucosa treated with DMBA, demonstrating a prominent granular layer underlying a mature, uniform stratum corneum. There are pleomorphism and alteration of the basal layer. Several mitotic figures are present. (H \& E stain; orig. mag. $\times 128$.)

association of neoplastic alteration with the use of the 20 per cent podophyllin.

Although carcinomatous changes were readily apparent in the mucosa treated with the carcinogenic agent, no malignant alterations were demonstrated in the normal mucosa exposed only to either one of the keratolytic drugs used in the study. Clinically, after the application of 20 per cent salicylic acid to normal mucosa, a slight decrease of surface keratosis was obvious. This keratolytic activity, however, was not as significant as that observed after the application of salicylic acid to conditioned

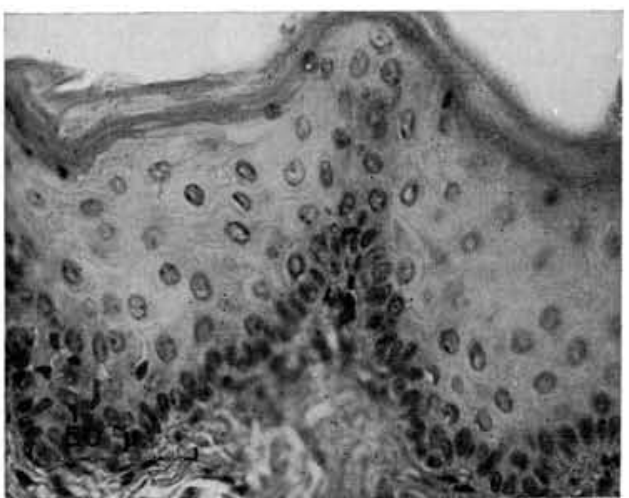

FIG. 2.-Salicylic acid applied to hamster oral mucosa conditioned with DMBA demonstrates acanthomatous changes with formation of large rete pegs. The stratum granulosum is absent and the keratin is without the normal compact laminated appearance. (H \& E stain; orig. mag. X128.) mucosa. Similar reactions of normal mucosa to 20 per cent podophyllin were not observed.

Microscopic observations.-After the application of 0.5 per cent DMBA to the mucosal epithelium, the stratum corneum became moderately thickened, and there was a generalized increase in keratinization of the prickle cell layer. The basal layer demonstrated focal pleomorphism, usually associated with disturbances in normal cell layering and disruption of layer integrity. Localized concentrations of inflammatory infiltrate in the corium overlying the striated muscle were associated with dyskeratotic changes in the epithelium (Fig. 1).

When the DMBA-conditioned mucosa was treated with 20 per cent salicylic acid, significant acanthosis and thickening of the epithelium resulted. The uniformly broadened stratum corneum, although lacking the laminated, compact appearance of untreated mucosal keratin, appeared granular and amorphous. No stratum lucidum was observed overlying the stratum granulosum which, when present, was thickened. Varying in its staining ability from a slight basophilia to a faint eosinophilia, the superficial prickle cell zone was homogeneous and relatively acellular. The residual cells in this region lacked definite membranes and nuclei. The cells of the basal layer were considerably elongated and basophilic, with focal disruption of their continuity. Multiple mitotic figures in association with dyskeratotic changes in the basal layer were observed overlying the region of subepithelial lymphocytic infiltration (Fig. 2).

Salicylic acid on normal mucosa produced a considerably thickened, acanthomatous epithelium that stained faintly. The stratum corneum, stratum lucidum, and granular layer exhibited the same characteristics observed in the tissue preconditioned with DMBA and treated with 20 per cent salicylic acid. The cells of the prickle layer below the stratum granulosum had highly keratinized cytoplasm. A generalized increase in inflammatory response in the corium was observed (Fig. 3).

When 20 per cent podophyllin was applied to mucosa pretreated with DMBA, little or no change, other than that produced by the carcinogenic agent alone, was observed. The basal layer, although appearing to be predominantly intact and normal, had 


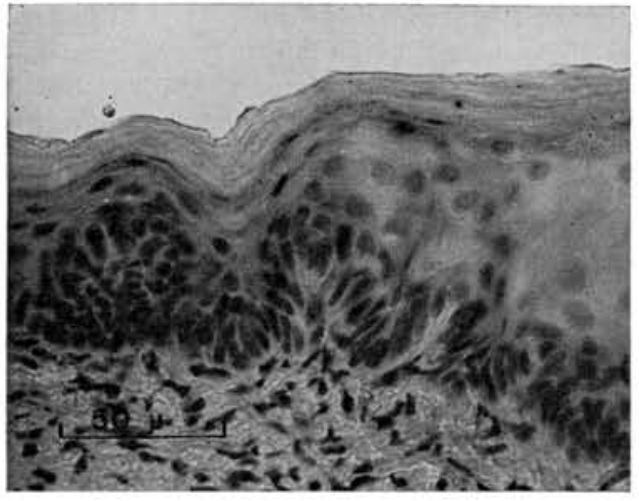

Fig. 3.-Salicylic acid applied to normal mucosal epithelium, demonstrating acanthomatous alterations with pronounced pleomorphism. The keratin layer is thickened and laminated, with underlying focal loss of stratum granulosum. The cells of the basal layer are elongated with focal proliferation. (H \& E stain; orig. mag. X128.)

focal dyskeratotic changes and large numbers of mitotic figures in late anaphase and telophase (Fig. 4).

The application of 20 per cent podophyllin to normal mucosa created no extensive alterations of the keratinization process. The most significant changes were observed in the basal layer, where there was an increased incidence of mitotic figures and concentration of pleomorphic proliferation (Fig. 5).

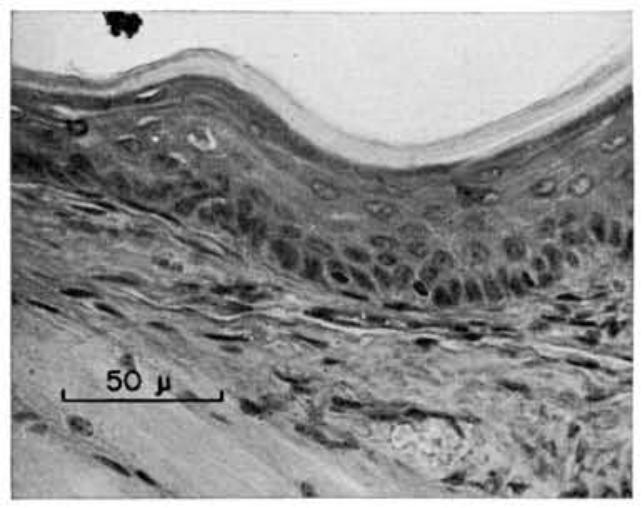

FIG. 4.-Hamster oral mucosa treated with podophyllin subsequent to the application of DMBA demonstrates relatively little alteration. The stratum corneum is uniform and mature, overlying a zone composed of chromatin granules from prickle cell breakdown. Cellular vacuolation is present in the prickle cell zone. (H \& E stain; orig. mag. $\times 128$.)

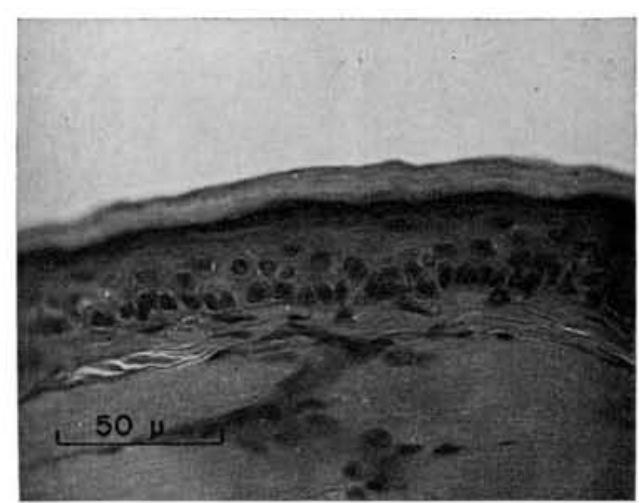

FIG. 5.-Normal hamster oral mucosa treated with podophyllin demonstrates a nonpapillated epithelium with a slightly thickened stratum corneum and a distinct granular layer. The basal layer is intact. (H \& E stain; orig. mag. $\times 128$.)

The topical application of only the vehicle, compound tincture of benzoin, to normal hamster oral mucosa produced no apparent changes. The histologic appearance resembled that of normal mucosa.

\section{Discussion}

The results obtained in this preliminary study varied considerably with the keratolytic agent employed. In the salicylic acid series, a decrease in the degree of oral keratosis was suggested clinically, even though comparable alterations were not observed microscopically. On the contrary, the amount of cornification actually appeared to have increased. The keratin observed after treatment with salicylic acid, however, lacked the compactness usually observed in the normal stratum corneum, acquiring instead a granular, amorphous appearance without the fibrous keratin laminations. This consistently observed change, compatible with a less opaque stratum corneum, was responsible for the clinical impression of decreased keratinization.

Salicylic acid-induced disturbances in the keratinization process are also well demonstrated by the abnormally formed or frequently absent stratum granulosum. The function of this zone is considered a distinct factor in epithelial keratinization by Mercer $^{11}$ and Rothman. ${ }^{12}$

In addition to its interference with the keratinization process, the salicylic acid elicited diffuse acanthomatous alterations, 
with elongation and broadening of the rete pegs in both the conditioned and normal mucosa. It seems apparent, therefore, that salicylic acid, in addition to its keratolytic properties, is a severe surface irritant. Although these findings are comparable in part to those reported by Sullivan and King, ${ }^{13}$ it is obvious that the mode of action of this agent involves more than a simple and direct fixing action.

Similar significant disruptions of the keratinization process were not observed in the podophyllin-treated mucosa. Although the stratum corneum was slightly increased in thickness, there was no apparent alteration in either the character of the keratin or the kera ${ }^{+}$ogenous zone.

In considering the relative ineffectiveness of 20 per cent podophyllin in the treatment or oral hyperkeratosis as observed in the study, its reported inability to penetrate the cornified epithelium ${ }^{2}$ should be noted. Although the degree of keratinization of the tissue was not excessive, the dense, compact, cornified state could conceivably have restricted the penetration of the agent as previously described by Nelson ${ }^{2}$ and Sullivan and King..$^{13}$ Since the response of the epithelium is dependent on the concentration of the drug and the physiologic state of the cells, however, epithelial resistance can be overcome by increasing the concentration of the agent. ${ }^{14}$ The period of application of the keratolytics was relatively short in this investigation due to extensive neoplastic involvement of the mucosa, so the effects of a longer period of treatment should be investigated. Moreover, since so little reaction was observed in the preconditioned epithelium and submucosa after 6 weeks of therapy with 20 per cent podophyllin, some degree of tissue tolerance may have been acquired. In such a situation, increased concentrations of the drug in addition to prolonged periods of application may be necessary for effective keratolysis.

In further attempting to explain the inert behavior of the topically applied podophyllin, attention should be directed toward the hydrogen-ion concentrations of the salivary secretions of the experimental hamsters. According to a study reported by Johansen and Rogosa,${ }^{15}$ the average salivary $\mathrm{pH}$ of Syrian hamsters fed a stock diet was 8.5. As previously reported by Viehover ${ }^{16}$ and later by Nelson, ${ }^{2}$ the therapeutic effec- tiveness of podophyllin is inactivated by alkaline solutions, the agent being converted to picropodophyllin and podophyllic acid.

Although the application of podophyllin to the hamster oral mucosa in this study was not impressive, its activity in a less alkaline medium should be investigated. Human salivary secretions vary from weakly acid to slightly alkaline ( $\mathrm{pH}$ ranging from 6.0 to 7.9) with an optimum $\mathrm{pH}$ of $6.6 .^{17}$

\section{Summary}

Two known keratolytic drugs, salicylic acid and podophyllin, were applied in 20 per cent solutions to induced oral hyperkeratotic lesions and to normal oral mucosa of 15 Syrian albino hamsters. After the initial gross alterations of desquamation, ulceration, and bleeding subsequent to the application of salicylic acid, a significant decrease in the amount of keratosis was clinically apparent. Alterations of the keratinization process during salicylic acid therapy were manifested as formation of a less opaque keratin layer and a disrupted stratum granulosum. In podophyllin-treated mucosa, no significant keratolytic effect was observed clinically or microscopically. Alterations of the keratinization process, comparable to those elicited by salicylic acid, were not obtained in podophyllintreated mucosa. The relative ineffectiveness of podophyllin in this study may have been due to its inability to penetrate the cornified epithelium, or due to inactivation by the alkaline saliva of the hamster.

The authors acknowledge the assistance of Dr. Donald Kerr, Professor of Oral Pathology, University of Michigan School of Dentistry, in the preparation and interpretation of the histologic sections.

\section{References}

1. Kaplan, I. W. Condylomata Acuminata. New Orleans med. surg. $J ., 94: 388-90,1942$.

2. Nelson, L. M. The Use of Podophyllum in Dermatology. Arch. Dermal. E* Syph., 67:488-95, 1953.

3. Walz, D. V. Podophyllin Treatment of Plantar Warts. Arch. Dermat. \& Syph., 65:490, 1952.

4. Shannon, J., and Sagher, F. Podophyllin Treatment in Various Skin Diseases. Dermatologica, 111:319-27, 1955.

5. Standish, S. M., and Moorman, W. C. Treatment of Hairy Tongue with Podophyllin Resin. J. Amer. dent. Ass., 68:535-40, 1964.

6. Winer, L. H. Black Hair Tongue: A Comparative Study of Black Hair Tongue, Geographic Tongue and a Drug Eruption of the Tongue. Arch. Dermat., 77: $97-103,1958$. 
7. Morris, A. L. Experimental Premalignant and Early Malignant Oral Epithelium in the Syrian Hamster. University of Rochester Dental Research Fellowship Program Proceedings, 1955, p. 84.

8. SAlLEY, J. J. Experimental Oral Carcinogenesis. University of Rochester Dental Research Fellowship Program Proceedings, 1955, p. 136.

9. Morris, A. L. Factors Influencing Experimental Carcinogenesis in the Hamster Cheek Pouch, $J$, deni. Res., 40:3 -15, 1961.

10. Marks, M. M. Condyloma Acuminatum: Podophyllin in Compound Tincture of Benzoin an Improvement in Technique of Treatment, J. Missouri med. Ass., 44:749, 1947.

11. MERCER, E. H. Keratin and Keratinization, International Series on Pure and Applied Biology. London, Pergamon Press, Ltd., 1961, p. 228-33.

12. Rothman, S. Physiology of Keratinization, Oral Surg., Oral Med. Oral Path., 7:1085-90, 1954.
13. Sullivan, M., and Krng, L. A. Effects of Resin on Podophyllum on Normal Skin, Condylomata Acuminata, and Verrucae Vulgaris, Arch. Dermat. \&o Syph., $56: 30-47,1947$.

14. King, L. S., and Cauldowele, E. W. Effects of Podophyllin on Mouse Skin. II. Consideration of Some Functional Aspects. $J$. nal. cancer Inst., 10: 131-46, 1949-50.

15. Johansen, E., and Rogosa, M. Determination of Oral pH in Syrian Hamsters, J. dent. Res., 32:702-03, 1953.

16. Viehover, A., and MACK, H. Biochemistry of the May Apple Root (Podophyllum peltatum), J. Amer. pharm. Ass. $27: 632-43,1938$.

17. Hawk, P. B., Oser, B. L., and Summerson, W. H Practical Physiological Chemistry, ed. 13. New York, The Blakiston Company, 1954, p. 350. 